\title{
An die Bezieher des „Landwirtschaftlichen Zentralblattes“
}

Entsprechend der zunehmenden Bedeutung des ,Landwirtschaftlichen Zentralblattes" haben Redaktion und Verlag die Zahl der Referate ständig erhöht und somit die Umfänge in den letzten Jahren wiederholt erweitert. Trotz dieser entstandenen zusätzlichen Kosten wurde der ursprüngliche Heftpreis unverändert zugunsten der Bezieher beibehalten. Es macht sich nunmehr aber erforderlich, eine realere Preisgestaltung anzustreben. Dabei ist es der Wunsch der Redaktion und des Verlages, die Abonnenten so wenig wie möglich zu belasten. Nachdem zahlreiche Bezieher des ,Landwirtschaftlichen Zentralblattes" darauf aufmerksam gemacht haben, daß sie die Schnelldokumentation, die den Abteilungen I und III seit 1961 beiliegt, nicht auswerten, haben wir uns entschlossen, ab Januar 1963 die Schnelldokumentation entfallen zu lassen. Der Heftpreis der Abteilungen I bis III bleibt jedoch unveründert 20,- DM.

$$
\text { Akademie-Verlag Redaktion }
$$


